\title{
Transition of Chytrid Fungus Infection from Mouthparts to Hind Limbs During Amphibian Metamorphosis
}

\author{
Taegan A. McMahon ${ }^{1}$ and Jason R. Rohr ${ }^{2}$ \\ ${ }^{1}$ Department of Biology, University of Tampa, 401 W. Kennedy Ave, Tampa, FL 33606 \\ ${ }^{2}$ Department of Integrative Biology, University of South Florida, Tampa, FL 33620
}

\begin{abstract}
The chytrid fungus, Batrachochytrium dendrobatidis (Bd), is implicated in worldwide amphibian declines. Bd has been shown to qualitatively transition from the mouthparts of tadpoles to the hindlimbs during metamorphosis, but we lack evidence of consistency in the timing of this transition across amphibian species. We also do not have predictive functions for the abundance of $\mathrm{Bd}$ in mouthparts and limbs as tadpoles develop or for the relationship between keratin and Bd abundance. Hence, researchers presently have little guidance on where to sample developing amphibians to maximize Bd detection, which could affect the accuracy of prevalence and abundance estimates for this deadly pathogen. Here, we show consistency in the timing of the transition of Bd from mouthparts to hind limbs across two frog species (Osteopilus septentrionalis and Mixophyes fasciolatus). Keratin and $\mathrm{Bd}$ simultaneously declined from the mouthparts starting at approximately Gosner stage 40. However, keratin on the hindlimbs began to appear at approximately stage 38 but, on average, Bd was not detectable on the hindlimbs until approximately stage 40, suggesting a lag between keratin and Bd arrival. Predictive functions for the relationships between developmental stage and keratin and developmental stage and $\mathrm{Bd}$ for mouthparts and hind limbs are provided so that researchers can optimize sampling designs and minimize erroneous conclusions associated with missing Bd infections or misestimating Bd abundance.
\end{abstract}

Keywords: Batrachochytrium dendrobatidis, amphibian decline, chytridiomycosis, quantitative PCR

The pathogenic chytrid fungus, Batrachochytrium dendrobatidis $(\mathrm{Bd})$, is associated with the extinction and extirpation of hundreds of amphibian species. Its distribution and transmission appear to be affected by several factors (Venesky et al. 2013), including the densities of amphibian and non-amphibian hosts (Briggs et al. 2010; Vredenburg et al. 2010; McMahon et al. 2013a), climate (Rohr et al. 2008; Rohr and Raffel 2010; Liu et al. 2013; Raffel et al.

Published online: November 11, 2014

Correspondence to: Taegan A. McMahon, e-mail: tmcmahon@ut.edu
2013), habitat (Raffel et al. 2010; Becker and Zamudio 2011; Murray et al. 2011; Liu et al. 2013), and pollution (McMahon et al. 2013b). Because of the devastating effects of Bd on amphibians, several researchers have suggested that $\mathrm{Bd}$ and amphibians require zealous management action (Woodhams et al. 2011; Venesky et al. 2012).

Bd infects keratinized or keratinizing tissue of amphibians (Altig 2007; Voyles et al. 2011), which are found in the mouthparts of tadpoles and the skin of metamorphic and adult frogs (Fellers et al. 2001). Marantelli et al. (2004) demonstrated that, as tadpoles approach 
metamorphosis, keratin degrades from tadpole mouthparts and develops on the hind limbs and Bd transitions from the mouthparts to the hind limbs. In this seminal study, however, Marantelli et al. (2004) only quantified Bd presence and absence on a single species using histology. Hence, it is unknown whether the timing of the transition of Bd from mouthparts to hindlimbs is consistent across amphibian species. Additionally, we also are without predictive functions for the prevalence and abundance of $\mathrm{Bd}$ (average number of Bd zoospores on infected and uninfected hosts) in mouthparts and limbs as tadpoles develop and for the relationship between keratin and Bd abundance. Knowing where Bd is located on the body at different points in development, especially near and through metamorphosis, should facilitate optimizing sampling design and efficiency. If researchers sample the wrong body location as Bd transitions from mouthparts to hind limbs, they could possibly miss infections completely or misestimate Bd abundance. This could result in erroneous conclusions, misconceptions, and lost time and money.

Here, we exposed Osteopilus septentrionalis tadpoles that varied in Gosner stage to Bd and then quantified Bd abundance in both the mouthparts and hind limbs of the same individuals. We then compared the timing of the transition of $\mathrm{Bd}$ from mouthparts to hind limbs in O. septentrionalis to Mixophyes fasciolatus, the species studied by Marantelli et al. (2004). We fit functions to the data to characterize the relationships among keratin, Bd, and developmental stage for the mouthparts and hind limbs of both species. We hypothesized that the two species would show similar timing for the transition of $\mathrm{Bd}$ from their mouthparts to hind limbs and that the decline of $\mathrm{Bd}$ in the mouthparts would occur simultaneously with the decline of mouthpart keratin. However, given that the keratin in the hindlimbs must develop before the $\mathrm{Bd}$ can infect and that it should take time for Bd in the mouthparts to locate keratin in the hindlimbs, we predicted a lag in the relationship between keratin and $\mathrm{Bd}$ on the hindlimbs. Once Bd arrives in the hindlimbs, we expected exponential Bd growth because of the abundant keratin resources.

In 2009, 35 O. septentrionalis tadpoles, from 5 mixed clutches, were collected from the University of South Florida campus (Gosner stages 25-28; Gosner 1960). Bd inoculum was prepared by growing $1 \mathrm{~mL}$ of Bd stock (strain SRS 812 isolated from Rana catesbeiana [Lithobates catesbeianus]) on $1 \%$ tryptone agar plates for 8 days at $23^{\circ} \mathrm{C}$. Each plate was flooded with $3 \mathrm{~mL}$ of deionized water and the water from each plate was homogenized. This solution was passed through a $20-\mu \mathrm{m}$ nylon filter (Spectrum Laboratories, Inc., Rancho Dominguez, CA) to isolate infective zoospores $\left(3 \times 10^{4}\right.$ zoospores $/ \mathrm{mL}$, counted on a hemocytometer).

Individual O. septentrionalis was exposed to $2 \mathrm{~mL}$ of the zoospore inoculum in $80 \mathrm{~mL}$ of artificial spring water (ASW; Cohen et al. 1980) for 2 days. Each O. septentrionalis and the inoculated water were then transferred to 1-L cups filled with fresh ASW and the frogs were maintained on a 12-h light cycle at $23^{\circ} \mathrm{C}$ and fed organic spinach ad libitum. Survival was monitored daily for 14 days, after which the animals were euthanized with an overdose of MS-222, Gosner stage was quantified, and mouthparts and one hind limb were removed using sterile scissors (scissors were sterilized after each tissue extraction) and preserved in separate vials containing $70 \%$ ethanol. To prevent crosscontamination of Bd DNA during handling, vinyl gloves used to handle each frog were rinsed sequentially in $10 \%$ bleach, $1 \%$ Novaqua $^{\circledR}$ to neutralize the bleach, and deionized water before handling the next frog.

We quantified Bd from amphibian mouthparts and hind limbs using the quantitative PCR ( $\mathrm{qPCR}$ ) procedures described by Kriger et al. (2006a, b). Samples were run once as recommended by Kriger et al. (2006a), and we added TaqMan ${ }^{\circledR}$ Exogenous Internal Positive Control (Exo IPC) Reagents (Applied Biosystems, Foster City, CA) to every reaction well to assess inhibition of the qPCR reaction (Kriger et al. 2006b). All samples were analyzed initially with a 1:100 dilution, and no samples were inhibited.

In our statistical analyses, we only included animals that had Bd detected on at least one of the two sampled body parts $(n=30)$. For the species M. fasciolatus, data were obtained from Marantelli et al. (2004). Keratin data are only available for $M$. fasciolatus. Statistics were analyzed with R statistical software (R Development Core Team 2010).

We first conducted logistic regression analyses on the $M$. fasciolatus data to generate predictive functions for the relationship between Gosner developmental stage and keratin and Bd presence. For the O. septentrionalis data, we generated predictive functions for the relationship between Gosner developmental stage and keratin presence (keratin data was from $M$. fasciolatus) using logistic regression and for developmental stage and Bd abundance using the following non-linear equation:

$$
\log _{10} \mathrm{Bd}=\mathrm{a} /\left(1+\exp \left(-\mathrm{b}^{\star}(\text { Gosner stage }-\mathrm{c})\right)\right) .
$$

The logistic models for $M$. fasciolatus revealed that nearly $0 \%$ of the tadpoles were predicted to have keratin on 

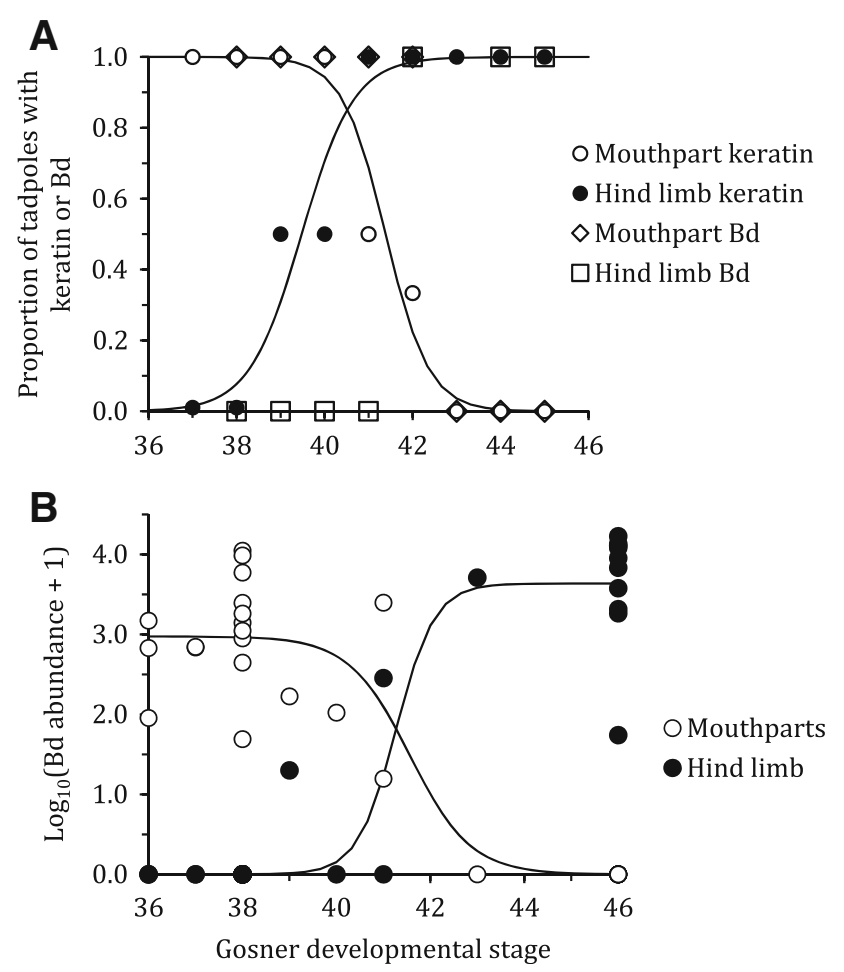

Fig. 1. The relationship between Gosner developmental stage and keratin and Batrachochytrium dendrobatidis (Bd) prevalence in the mouthparts and hind limbs of the tadpole Myophyes fasciolatus (a), and the relationship between Gosner developmental stage and $\mathrm{Bd}$ abundance (zoospore genome equivalents) in the mouthparts and hind limbs of the tadpole O. septentrionalis $(n=30)$ (b). For $M$. fasciolatus, the logistic regression best-fit curves are for the keratin data. Logistic regression could not be successfully conducted on the $\mathrm{Bd}$ data for this species because there was too little variation (all the proportions were either 0 or 1). Data for $M$. fasciolatus were acquired from Marantelli et al. (2004)

their hind limbs at stage 37 , whereas $\sim 100 \%$ were predicted to have keratin on their hind limbs by stage 42 (intercept $\pm \mathrm{SE}=-66.224 \pm 40.096$, slope $\pm \mathrm{SE}=$ $1.677 \pm 1.013$; Fig. 1a). Keratin on M. fasciolatus began to decline in the mouthparts well after it began appearing in the hind limbs (2004). Nearly, 100\% of the tadpoles were predicted to have keratin on their mouthparts at stage 39 , whereas $\sim 0 \%$ were predicted to have keratin on their mouthparts by stage 44 (intercept $\pm \mathrm{SE}=84.241 \pm 45.414$, slope $\pm \mathrm{SE}=-2.035 \pm$ 1.095; Fig. 1a). The Bd prevalence data generally matched the location of keratin (Fig. 1a). We could not fit logistic functions to the prevalence data because of the lack of variability (all proportions were 0 or 1 ). However, what is striking is that $\mathrm{Bd}$ in the mouthparts seems to decline concurrently with the decline in mouthpart keratin but appears on the hind limbs 2-3 Gosner stages after keratin is detected there (Fig. 1a).

The non-linear models fit the O. septentrionalis data well for both the mouthparts $(\mathrm{a} \pm \mathrm{SE}=2.97452 \pm 0.15857$, $\mathrm{b} \pm \mathrm{SE}=-1.53965 \pm 1.05962, \mathrm{c} \pm \mathrm{SE}=41.56376 \pm 0.51600$; Fig. 1b) and hind limbs $(\mathrm{a} \pm \mathrm{SE}=3.63493 \pm$ $0.18411, \mathrm{~b} \pm \mathrm{SE}=2.45352 \pm 3.31990, \mathrm{c} \pm \mathrm{SE}=41.27424 \pm$ 0.45164; Fig. 1b), accounting for 85.8 and $89.7 \%$ of the variation, respectively. The timing of the transition of $\mathrm{Bd}$ in $O$. septentrionalis from the mouthparts to the hind limbs generally matched the timing of the transition in M. fasciolatus (Fig. 1a, b). For both species, the decline of $\mathrm{Bd}$ in the mouthparts generally occurred between stages 40 and 44 and Bd increased on the hind limbs between stages 40 and 43 , despite keratin appearing on the hindlimbs of $M$. fasciolatus between stages 38 and 41 (Fig. 1).

There was substantial time when both the mouthparts and hind limbs had keratin (Fig. 2a), but much smaller overlap when they both had Bd (Fig. 2b). Plotting the predicted values of keratin versus $\mathrm{Bd}$ abundance as functions of Gosner stage provides the most salient view of these dynamics. As predicted, a decline in mouthpart keratin caused an immediate loss of mouthpart Bd as reflected by the tight linear relationship between the predicted values for mouthpart keratin and Bd abundance on the mouthparts $\left(y=9.7327 x, R^{2}=0.996\right.$; Fig. $\left.3 \mathrm{a}\right)$. In contrast and also as predicted, the keratin was present on the hind limbs for some time before Bd arrived and then grew exponentially ( $y=1 \mathrm{E}-04 \mathrm{e}^{34.697 x}$, Fig. 3b). Indeed, the exponential fit accounted for $96.6 \%$ of the variation between the predicted values for hind limbs keratin and Bd abundance on the hind limbs (Fig. 3b).

We show that $M$. fasciolatus and O. septentrionalis tadpoles, which occupy two continents, exhibit almost identical timing in the transition of $\mathrm{Bd}$ from their mouthparts to their hind limbs as a function of Gosner developmental stage. Moreover, the amount of keratin in the mouthparts and hind limbs of $M$. fasciolatus was highly predictive of the transition of Bd from the mouthparts to the hind limbs of $O$. septentrionalis. These results suggest that the transition of keratin from the mouthparts to the hind limbs is likely a conserved trait of tadpole development that drives the shift in body location of Bd infections. However, examining these patterns in more than two species will be necessary to better evaluate the generality of our findings. 

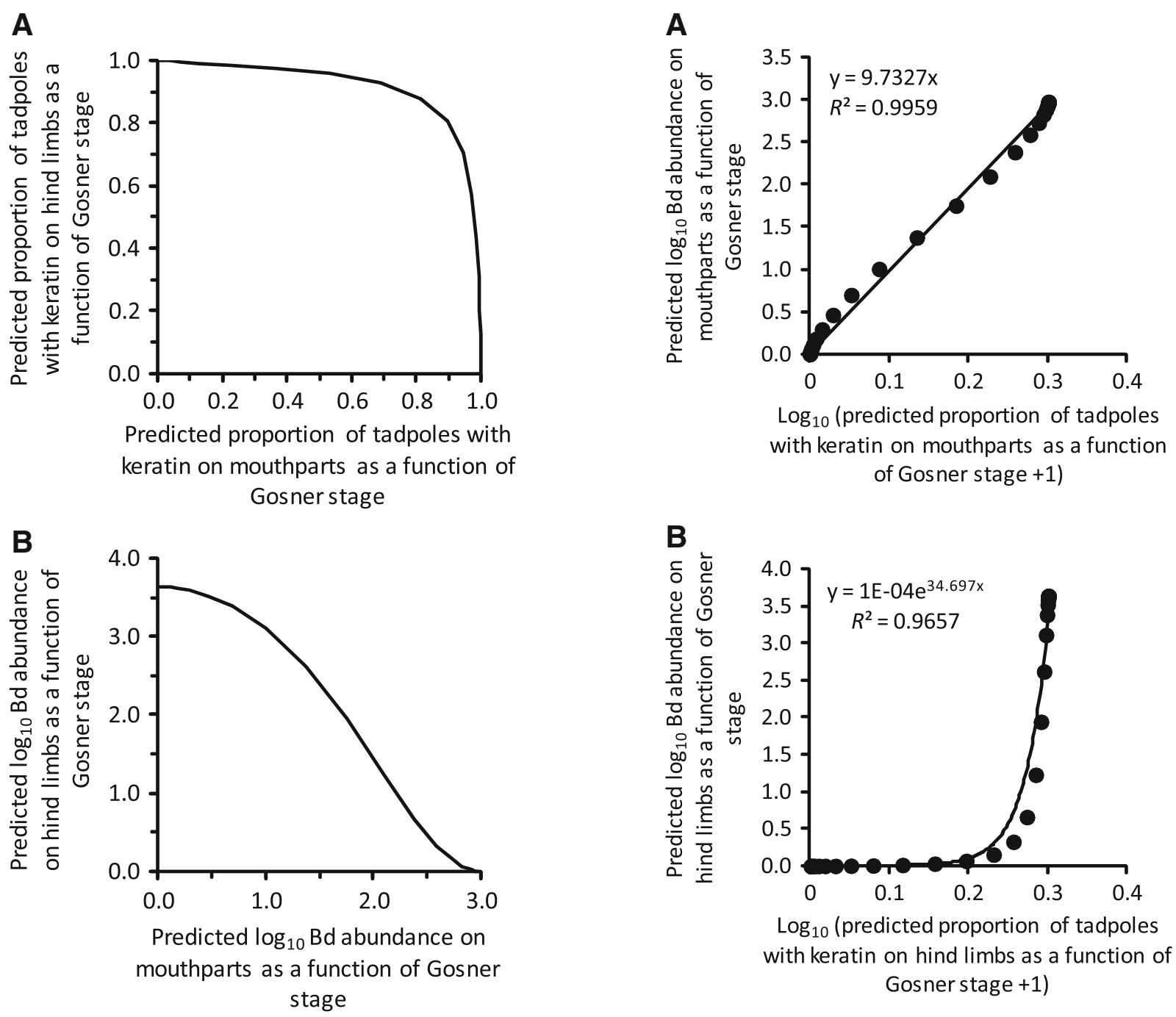

Fig. 2. Relationships between the predicted values for keratin on tadpole mouthparts and hindlimbs as a function of Gosner developmental stage (a) and for $\mathrm{Bd}$ abundance on tadpole mouthparts and hind limbs as a function of Gosner developmental stage (b)

As predicted, the loss of keratin in the mouthparts was associated with a concurrent loss of $\mathrm{Bd}$ there. This is probably because $\mathrm{Bd}$ is at some equilibrium level, and the loss of resources and substrate directly reduces the amount of Bd that can be supported. In contrast, the development of keratin on the hind limbs does not simultaneously result in Bd detection there. This is likely because zoospores from the mouthparts need to (1) locate the new keratin on the hind limbs, (2) successfully infect the hind limbs, and (3) replicate sufficiently for the swabbing and qPCR to detect the $\mathrm{Bd}$. On average, there appears to be a delay of approximately 2-3 Gosner stages between keratin on the

Fig. 3. Relationships between the predicted values for keratin and Bd abundance on tadpole mouthparts as a function of Gosner developmental stage (a) and for keratin and Bd abundance on tadpole hind limbs as a function of Gosner developmental stage (b). Shown are best-fit lines, best-fit equations, and the proportion of variance for which the equation accounts

hind limbs and $\mathrm{Bd}$ detection. Once $\mathrm{Bd}$ arrives, the abundance, as expected, fits an exponential growth function.

Understanding the transition of Bd from the mouthparts to the limbs is crucial to preventing misconceptions. For example, if we sampled only the mouthparts, we could have erroneously concluded that tadpoles lost their infections during metamorphosis. Alternatively, if we sampled only limbs, we could have erroneously concluded that tadpoles were more resistant to $\mathrm{Bd}$ than metamorphs. Additionally, prevalence and Bd loads would have been misestimated if we only sampled one of the two body locations. Importantly, several studies have documented 
presumed costs of exposure to $\mathrm{Bd}$ in the absence of either detectable infections or sufficient time for Bd to reach densities high enough to typically cause pathology (e.g., see: Blaustein et al. 2005; Garner et al. 2009; Venesky et al. 2009; Luquet et al. 2012). However, it is unclear in these experiments whether the authors simply missed infections by sampling the wrong body locations. This is critical because we now have confirmed evidence that $\mathrm{Bd}$ can release a chemical that can cause pathology in the absence of infections (McMahon et al. 2013a, 2013b). Missed infections because of sampling the wrong body location may have also led to unpublished research. Given the findings of this study, it might be worth re-sampling preserved amphibian specimens.

In summary, we recommend that researchers sample both the mouthparts and limbs of amphibians if they expect to be taking $\mathrm{Bd}$ measurements from amphibians between Gosner stages 38 and 46. If this is cost prohibitive and assuming our results are consistent across species, we recommend using the provided functions to estimate where on the body is the best location to sample. A crude recommendation based on these functions is to sample the mouthparts of amphibians less than Gosner stage 41 and the hind limbs of amphibians greater than Gosner stage 41 . The provided functions should offer the best chances of accurately determining if there is a Bd infection present and the true abundance of the infection. These recommendations should increase sampling efficiency, diminish the likelihood that infections will be missed, reduce the likelihood of drawing erroneous conclusions, might reduce qPCR costs ( $>\$ 25 /$ sample if done in triplicate and labor is included), and should help ensure that limited conservation resources are used wisely.

\section{ACKNOWLEDGMENTS}

We thank V. Vasquez for providing the $B d$. Funds were provided by Grants from the National Science Foundation (DEB 0516227 and IOS-1121758), the US Department of Agriculture (NRI 2006-01370 and 2009-35102-0543), and the US Environmental Protection Agency STAR Grant R833835).

\section{REFERENCES}

Altig R (2007) Comments on the descriptions and evaluations of tadpole anomalies. Herpetological Conservation and Biology $2: 1-4$
Becker CG, Zamudio KR (2011) Tropical amphibian populations experience higher disease risk in natural habitats. Proceedings of the National Academy of Sciences of the United States of America 108:9893-9898

Blaustein AR, Romansic JM, Scheessele EA, Han BA, Pessier AP, Longcore JE (2005) Interspecific variation in susceptibility of frog tadpoles to the pathogenic fungus Batracbochytrium dendrobatidis. Conservation Biology 19:1460-1468

Briggs CJ, Knapp RA, Vredenburg VT (2010) Enzootic and epizootic dynamics of the chytrid fungal pathogen of amphibians. Proceedings of the National Academy of Sciences of the United States of America 107:9695-9700

Cohen LM, Neimark H, Eveland LK (1980) Schistomsoma mansoni: response of cercariae to a thermal gradient. Journal of Parasitology 66:362-364

Fellers GM, Green DE, Longcore JE (2001) Oral chytridiomycosis in the mountain yellow-legged frog (Rana muscosa). Copeia 4:945-953

Garner TW, Walker S, Bosch J, Leech S, Rowcliffe JM, Cunningham AA, et al. (2009) Life history tradeoffs influence mortality associated with the amphibian pathogen Batrachochytrium dendrobatidis. Oikos 118:783-791

Gosner KL (1960) A simplified table for staging anuran embryos and larvae with notes on identification. Herpetologica 16:183190

Kriger KM, Hero JM, Ashton KJ (2006) Cost efficiency in the detection of chytridiomycosis using PCR assay. Diseases of Aquatic Organisms 71:149-154

Kriger KM, Hines HB, Hyatt AD, Boyle DG, Hero JM (2006) Techniques for detecting chytridiomycosis in wild frogs: comparing histology with real-time Taqman PCR. Diseases of Aquatic Organisms 71:141-148

Liu X, Rohr JR, Li YM (2013) Climate, vegetation, introduced hosts and trade shape a global wildlife pandemic. Proceedings of the Royal Society B-Biological Sciences 280:20122506

Luquet E, Garner TWJ, Léna J-P, Bruel C, Joly P, Lengagne T, et al. (2012) Genetic erosion in wild populations makes resistance to a pathogen more costly. The Society for the Study of Evolution 66:1942-1952

Marantelli G, Berger L, Speare R, Keegan L (2004) Distribution of the amphibian chytrid Batrachochytrium dendrobatidis and keratin during tadpole development. Pacific Conservation Biology 10:173-179

McMahon TA, Brannelly LA, Chatfield MWH, Johnson PTJJoseph MB, McKenzie VJ, et al. (2013) Chytrid fungus Batrachochytrium dendrobatidis has nonamphibian hosts and releases chemicals that cause pathology in the absence of infection. Proceedings of the National Academy of Science 110:210-215

McMahon TA, Romansic JM, Rohr JR (2013) Non-monotonic and monotonic effects of pesticides on the pathogenic fungus Batrachochytrium dendrobatidis in culture and on tadpoles. Environmental Science and Technology 47:7958-7964

Murray KA, Retallick RWR, Puschendorf R, Skerratt LF, Rosauer D, McCallum HI, et al. (2011) Assessing spatial patterns of disease risk to biodiversity: implications for the management of the amphibian pathogen, Batrachochytrium dendrobatidis. Journal of Applied Ecology 48:163-173

R Development Core Team (2010) R: A language and environment for statistical computing. R Foundation for Statistical Computing Version 2.8.1.

Raffel TR, Halstead NT, McMahon T, Romansic JM, Venesky MD, Rohr JR (2013) Disease and thermal acclimation in a more 
variable and unpredictable climate. Nature Climate Change 3:146-151

Raffel TR, Michel PJ, Sites EW, Rohr JR (2010) What drives chytrid infections in newt populations? Associations with substrate, temperature, and shade EcoHealth 7:526-536

Rohr JR, Raffel TR (2010) Linking global climate and temperature variability to widespread amphibian declines putatively caused by disease. Proceedings of the National Academy of Sciences of the United States of America 107:8269-8274

Rohr JR, Raffel TR, Romansic JM, McCallum H, Hudson PJ (2008) Evaluating the links between climate, disease spread, and amphibian declines. Proceedings of the National Academy of Sciences of the United States of America 105:17436-17441

Venesky MD, Mendelson JR, Sears BF, Stiling PD, Rohr JR (2012) Selecting for tolerance against pathogens and herbivores to enhance the success of reintroduction and translocation programs. Conservation Biology 26:586-592
Venesky MD, Parris MJ, Storfer A (2009) Impacts of Batrachochytrium dendrobatidis infection on tadpole foraging performance. Ecohealth 6:565-575

Venesky MD, Raffel TR, McMahon TA, Rohr JR (2013) Confronting inconsistencies in the amphibian-chytridiomycosis system: implications for disease management. Biological Reviews 89:477-483

Voyles J, Rosenblum EB, Berger L (2011) Interactions between Batrachochytrium dendrobatidis and its amphibian hosts: a review of pathogenesis and immunity. Microbes and Infection 13:25-32

Vredenburg VT, Knapp RA, Tunstall TS, Briggs CJ (2010) Dynamics of an emerging disease drive large-scale amphibian population extinctions. Proceedings of the National Academy of Sciences of the United States of America 107:9689-9694

Woodhams DC, Bosch J, Briggs CJ, Cashins S, Davis LR, Lauer A, Muths E, Puschendorf R, Schmidt BR, Sheafor B, et al. (2011) Mitigating amphibian disease: strategies to maintain wild populations and control chytridiomycosis. Frontiers in Zoology 8:8 . doi:10.1186/1742-9994-8-8 